\title{
Gastric duplication in an adult mimicking mucinous cystadenoma of the pancreas
}

\author{
X B D'Journo, V Moutardier, O Turrini, J Guiramand, B Lelong, C Pesenti, G Monges, \\ M Giovannini, J R Delpero
}

Gastric duplication cyst (GDC) in an adult can have several clinical presentations. A review of the literature showed previously reported cases of GDC presenting as pancreatic pseudocyst or with greatly raised concentrations of carbohydrate antigen 19-9 (CA 19-9). It is often difficult to discriminate GDC from pancreatic cystic tumour, in particular pancreatic mucinous cystadenoma, in which concentrations of carcinoembryonic antigen and CA 19-9 are classically raised. This report describes an adult case of GDC mimicking a mucinous cystadenoma of the pancreas. This is the first report of a simultaneous increase in carcinoembryonic antigen and CA 19-9 in GDC in the absence of malignancy. Although few cases of carcinoma arising from a GDC having been reported, the production of oncofetal antigens raises the problem of a precancerous condition in long standing intestinal duplications. In this situation surgical resection must be performed.

A gastric duplication cyst (GDC) in an adult is a rare entity because it is usually discovered early in life. This congenital anomaly, comprising $4 \%$ of all alimentary duplications, ${ }^{1}$ can have several clinical presentations. A review of the literature showed previously reported cases of GDC presenting as pancreatic pseudocyst, ${ }^{2}$ or with greatly raised concentrations of carbohydrate antigen 19-9 (CA 199). ${ }^{3}$ It is often difficult to discriminate GDC from pancreatic cystic tumours, in particular pancreatic mucinous cystadenoma, in which concentrations of carcinoembryonic antigen (CEA) and CA 19-9 are classically raised. ${ }^{4}$

\section{"It is offen difficult to discriminate gastric duplication cyst from pancreatic cystic tumours, in particular pancreatic mucinous cystadenoma"}

We report an adult case of GDC mimicking a mucinous cystadenoma of the pancreas. To our knowledge, this is the first report of a simultaneous rise in CEA and CA 19-9 in GDC in the absence of malignancy. Few cases of carcinoma arising from a GDC having been reported ${ }^{15}$; the production of an oncofetal antigen raises the problem of a precancerous condition in long standing intestinal duplications. In this situation surgical resection must be performed.

\section{CLINICAL PRESENTATION}

A healthy 29 year old white woman was admitted to our institution because of recurrent epigastric pain and unspecified lipothymia. Her medical history was unremarkable. Physical examination was normal. Ultrasound examination and computed tomography (CT) scan revealed a homogeneous cystic mass, measuring $8 \mathrm{~cm}$, in the epigastric region, in contact with the pancreas and the stomach (fig lA). Peripheral calcifications were noted on CT scan around the cyst. CT scan also revealed another cystic mass located near the spinal cord in the posterior mediastinum, which showed no evidence of malignancy (fig 1B). Endoscopic ultrasonography (EUS) revealed that the cystic mass had its own clear thin wall and an exploratory puncture was made. A diagnosis of GDC was suggested by EUS but the clinical presentation led us to make another diagnosis. The cyst contained a yellowish serous fluid, which was aspirated so that the concentrations of CEA and CA 19-9 could be measured. Although serum concentrations of CEA $(<0.5 \mu \mathrm{g} /$ litre $)$ and CA $19-9$ (17 kui/litre; normal range, < 35 kui/litre) were within the normal range before surgery, the contents of the cyst revealed very high concentrations of CEA (36 $050 \mu \mathrm{g}$ / litre) and CA 19-9 (659 510 kui/litre). Fluid cultures grew no bacteria and cytological examination of the fluid revealed scanty cellularity, with no malignant cells. A diagnosis of pancreatic mucinous cystadenoma was established with the following supporting arguments: young woman, morphological presentation, peripheral pancreatic calcifications, and very high CEA and CA 19-9 concentrations.

Laparotomy was performed because of the risk of malignancy. The lesion was identified on the greater curvature of the stomach (fig 2). The pancreas was strictly normal. The lesion was locally excised with a margin of normal tissue (fig 3). There was no communication with the lumen of the stomach. Pathological examination revealed strict morphological criteria for the diagnosis of gastric duplication cyst, ${ }^{1}$ namely: the cyst was attached to the
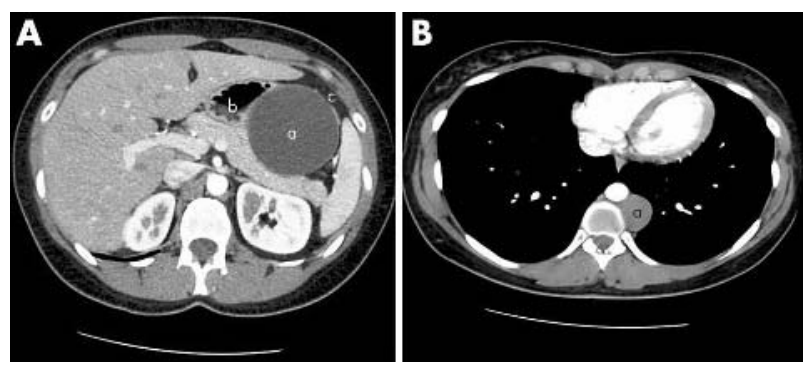

Figure 1 Computed tomography (CT) findings. (A) A diagnosis of pancreatic mucinous cystadenoma was established with the following supporting arguments: young woman, CT presentation (peripheral calcifications, own clear thin wall), and greatly raised concentrations of carcinoembryonic antigen and carbohydrate antigen 19-9 in the cyst (a, gastric duplication cyst; $b$, stomach; $c$, peripheral calcifications). (B) CT scan found another cystic mass (a) located near the spinal cord in the posterior mediastinum, which showed no signs of malignancy.

Abbreviations: $\mathrm{CA}$, carbohydrate antigen; $\mathrm{CEA}$, carcinoembryonic antigen; CK, cytokeratin; CT, computed tomography; EUS, endoscopic ultrasonography; GDC, gastric duplication cyst 


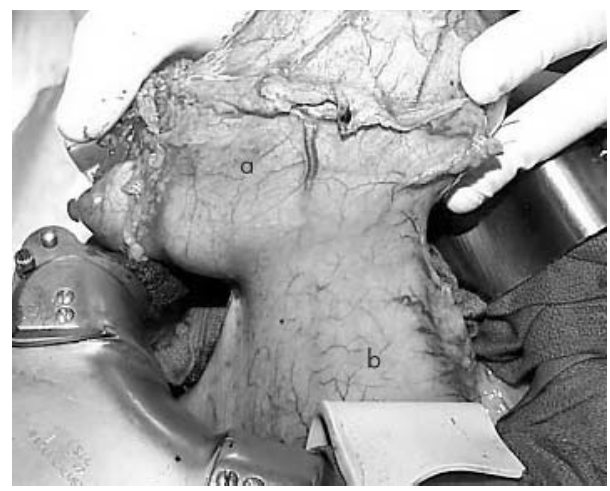

Figure 2 Gross pathology. The lesion was found on the greater curvature of the stomach. There was no communication between the lumen of the stomach and the cyst (a, gastric duplication cyst; b, stomach).

stomach and contiguous with its wall, the lesion was surrounded by at least one coat of smooth muscle fusing with the muscularis propria of the stomach, and the cyst was lined by typical gastric mucosa, accompanied by patches of ectopic or immature cells (figs 4-6). Although most of the epithelium was missing, some parts of the cyst contained simple cuboidal epithelium and ciliated pseudostratified epithelium, and these resembled normal mucosa of the alimentary tract at various embryonic stages (figs 7 and 8 ). Immature cells with eosinophilic cytoplasm and a round nucleus with large amounts of chromatin were seen in the gastric glands of the duplication cyst.

The pathological examination also revealed that the cyst comprised three non-communicating cysts: a main cyst measuring $6 \mathrm{~cm}$ and two accessory cysts measuring $1 \mathrm{~cm}$.

Immunohistochemical analysis revealed synaptophysin and chromogranin positive cells in the epithelium (fig 9), indicating that the gastric wall and the duplication wall had a normal endocrine component. GDC was immunopositive for cytokeratin 7 (CK7) and CK20. The gastric mucosa was immunopositive for CK20 but was immunonegative for CK7, suggesting that the epithelium of the GDC derived from the alimentary tract and contained some ectopic cells immunopositive for CK7. In fact, the anti-CK7 antibody reacts with proteins that are found in most ductal, glandular, and transitional epithelia of the urinary tract and bile duct epithelial cells. The GDC was immunopositive for CA 19-9 and weakly positive for CEA, whereas the gastric mucosa was immunonegative for both. The CA 19-9 and CEA antigens are highly expressed in gastrointestinal adenocarcinomas, although the pathological examination of the GDC found no sign of malignancy. A diagnosis of GDC was made. The postoperative outcome was uneventful and the patient was discharged.

\section{DISCUSSION}

Duplications of the intestinal tract can occur anywhere from the mouth to the anus. The duplication cyst is entirely separate from the adjacent bowel and shares a common wall. GDC comprises $4 \%$ of all alimentary duplications, ${ }^{1}$ and diagnosis is usually made early in life; approximately $67 \%$ of patients present within the lst year of life and it is a rare entity in adults. Strict morphological criteria have been established for the correct diagnosis of duplication cysts. The pathogenesis is controversial, but abnormal recanalisation after the solid epithelial stage of embryonic bowel development is thought by most to underlie this lesion. ${ }^{5}$ GDCs in adults are more common in female patients and can have several clinical presentations. It is often difficult to

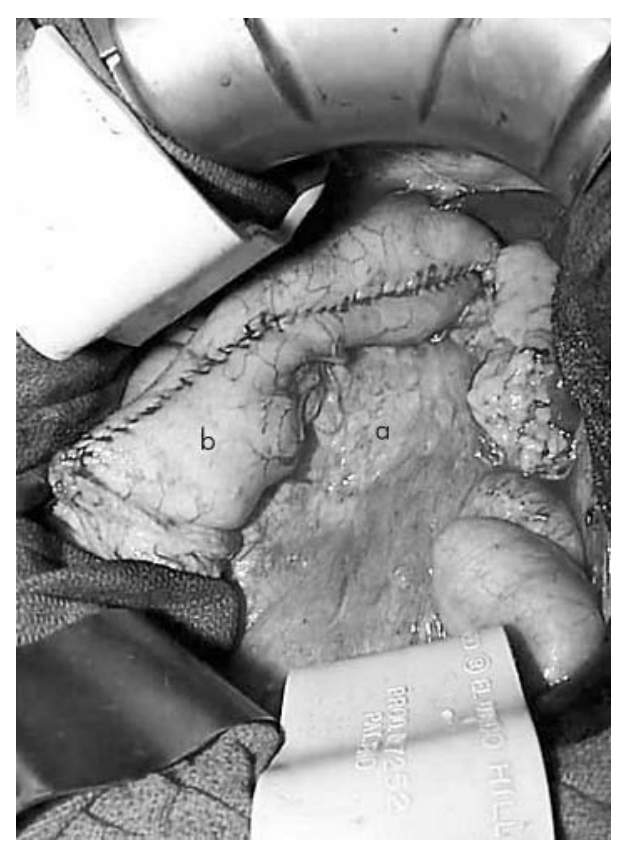

Figure 3 The lesion was locally excised with a margin of normal tissue. The pancreas was strictly normal ( $a$, pancreas; $b$, stomach).

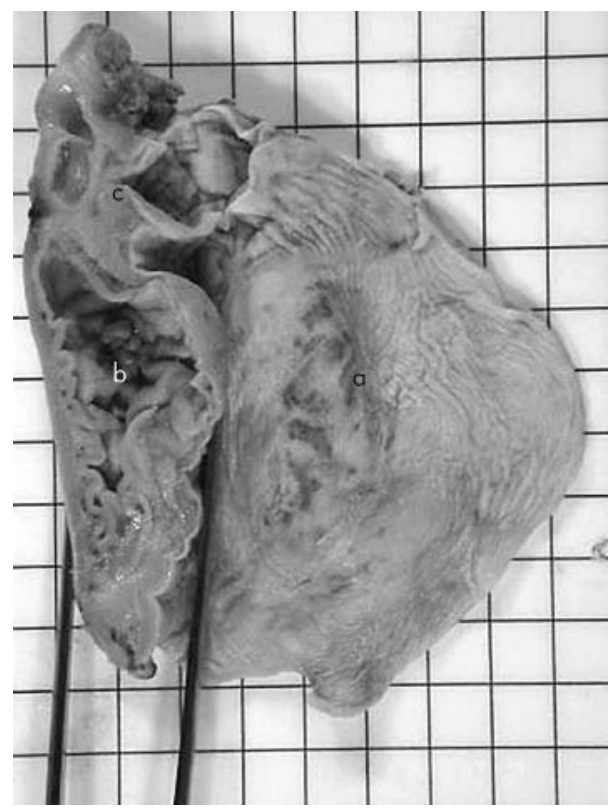

Figure 4 The cyst (a) was attached to the stomach (b) and contiguous with its wall. Otherwise, the pathological examination revealed that the cyst was made up of three separate non-communicating cysts (c).

discriminate GDC from congenital cysts or malignant pancreatic cystic tumours. Differential diagnoses must be considered. The presenting symptoms are non-specific and can be revealed by complications such as infection, haematemesis, compression, or carcinoma arising in the cyst.

GDC can mimic a pancreatic pseudocyst, and recurrent episodes of pancreatitis ${ }^{1}$ have been described, especially in those patients in whom the duplication is contiguous with the stomach. It is often difficult to discriminate GDC from malignant pancreatic cystic tumour, ${ }^{2}$ in particular pancreatic mucinous cystadenoma. The diagnosis of pancreatic mucinous cystadenoma is based on the clinical presentation, the 


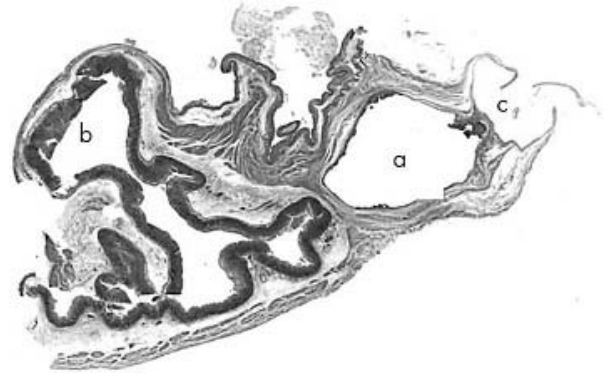

Figure 5 Pathological examination revealed the diagnosis of gastric duplication cyst (a): the cyst was attached to the stomach and contiguous with its wall, the lesion was surrounded by at least one coat of smooth muscle fusing with the muscularis propria of the stomach (b), and the cyst was lined by typical gastric mucosa, accompanied by patches of ectopic or immature cells. The cyst was made up of three separate noncommunicating cysts (c).

CT scan, EUS, and the high concentrations of CEA. The diagnosis must be confirmed by surgery and pathological examination. Because cytology is a relatively insensitive test, cyst fluid tumour markers such as CEA have been used to improve the sensitivity for the detection of malignancy. Cyst fluid CEA values are uniformly low in serous cystadenomas, higher in mucinous lesions, and very high in mucinous cystadenocarcinomas. ${ }^{4}$ In our report, the contents of the cyst revealed very high concentrations of CEA and CA 19-9, but pathological examination found no signs of malignancy. Very high concentrations of CA 19-9 have been described in GDC previously, ${ }^{3}$ and high concentrations of CA 19-9 and CEA have been reported in an adult ileal duplication cyst. ${ }^{6}$ To our knowledge, this is the first report of a simultaneous increase in CEA and CA 19-9 in GDC in the absence of malignancy.

The production of oncofetal antigens by the epithelial lining of alimentary tract duplication cysts in adults raises the problem of a precancerous condition in long standing intestinal duplications. Malignant transformation has been described in a few adult cases. ${ }^{15}$ Carcinoma arising from a duplication cyst is extremely rare, with only five cases reported in gastric duplication cysts to date. ${ }^{5}$ It is possible that immature epithelia produce oncofetal antigens, although we did not verify this hypothesis. Otherwise, immunohistochemical analysis (synaptophysin and chromogranin) indicated a normal endocrine component in the gastric wall and the duplication wall with no indication of malignancy. The production of oncofetal antigens is the result of overexpression of embryonic antigens in immature cells. Their abnormal secretion by gastric duplication cysts may be caused by

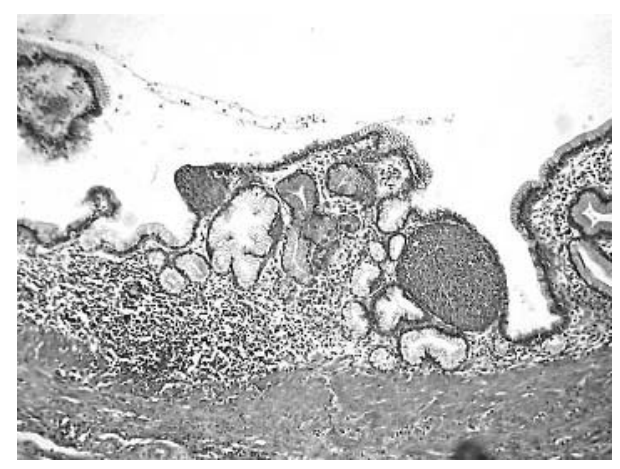

Figure 6 Microscopically, the cyst wall had a well circumscribed smooth muscle layer, which corresponded to the muscularis propria of the stomach, and the cyst was lined by typical gastric mucosa (original magnification, $\times 40$ ).

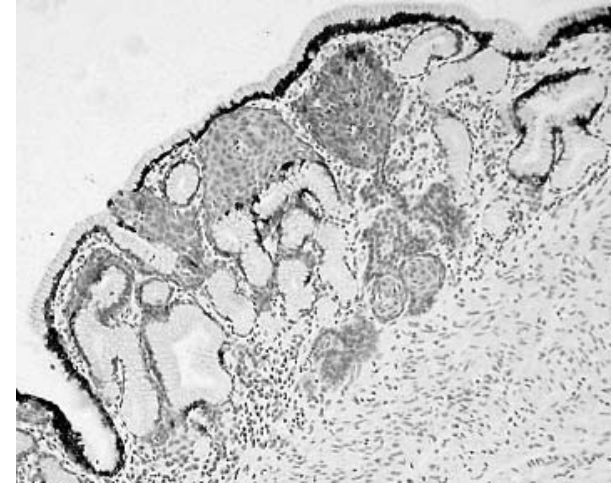

Figure 7 Although most of the epithelium was missing, parts of the cyst contained simple cuboidal epithelium and ciliated pseudostratified epithelium, and these resembled normal mucosa of the alimentary tract at various embryonic stages. No pathological criteria of malignancy were found (original magnification, $\times 100$ ).

environmental agents that probably lead to benign metaplasia in a long standing cyst. Benign metaplasia may signal the presence of a potentially carcinogenic microenvironment, whereas other types of metaplasia, such as incomplete intestinal metaplasia, are regarded as precancerous. Some reports have suggested that longterm benign cysts with raised tumour markers have a greater degenerative potential. ${ }^{7}$

\section{"Non-communicating gastric duplicating cyst is a rare entity in adults and it is difficult to discriminate from malignant pancreatic cystic tumour"}

Various other congenital anomalies are present in 35\% of gastric duplication cases. ${ }^{1}$ Alimentary duplication, oesophageal diverticulum, or spinal cord abnormalities are often encountered. Accordingly, in our case, a cystic mass with no signs of malignancy was located near the spinal cord in the posterior mediastinum. CT scans and EUS are the best way to identify GDC. Classically, radiographic examination shows an intramural filling defect indenting the gastric contour. GDCs have their own wall on CT scan, they are located along the greater curvature, and they have a cystic or tubular configuration. Sometimes the relation of these cysts with the neighbouring organs is difficult to specify. The imaging appearances of pancreatic cysts are well defined, but were indistinguishable from GDC on CT scan in our present report, namely: thin wall, calcifications, shape, size, location. Nevertheless, EUS enabled us to make a diagnosis of GDC

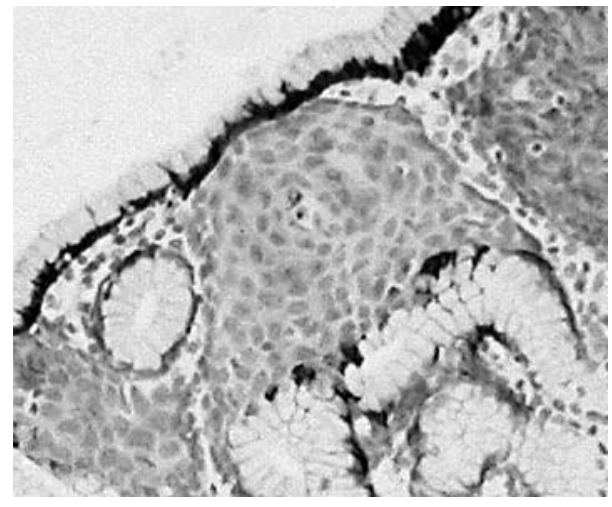

Figure 8 Immature cells with eosinophilic cytoplasm and a round nucleus with a large amount of chromatin were seen in the gastric glands of the duplication cyst (original magnification, $\times 200$ ). 


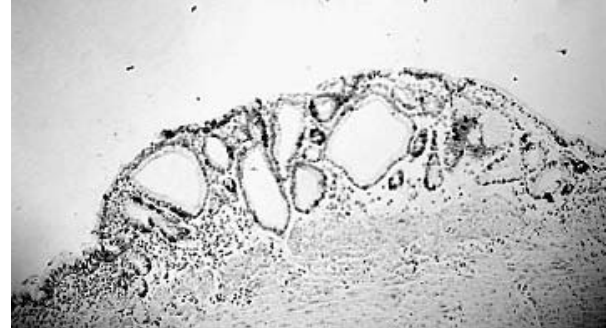

Figure 9 Immunohistochemical analysis revealed synaptophysin and chromogranin positive cells in the epithelium, indicating a normal endocrine component in the gastric wall and the duplication wall, with no criteria of malignancy (original magnification, $\times 100$ ).

and allowed us to complete the exploration by puncturing the cyst. Because malignant transformation has been described in a few adult cases, ${ }^{15}$ surgical removal is considered to be the best treatment. Non-communicating GDC is classically treated by complete resection, performing excision of the shared wall between the stomach and the duplication. Some recent studies reported successful laparoscopic resection, ${ }^{8}$ but in our case we performed laparotomy because a diagnosis of pancreatic mucinous cystadenoma was suspected before surgery. However, the size of the cyst required open surgery. Communicating GDC usually requires no intervention when both gastric lumens are patent.

Non-communicating GDC is a rare entity in adults and it is difficult to discriminate from malignant pancreatic cystic tumour. Classically, concentrations of CEA and CA 19-9 are raised in pancreatic mucinous cystadenoma, but only CA 199 is raised in GDC. To our knowledge, this is the first report of a simultaneous rise in CEA and CA 19-9 in GDC in the absence of malignancy. Few cases of carcinoma arising from a GDC having been reported, and the production of oncofetal antigens raises the problem of a precancerous condition in long standing intestinal duplications. In this situation surgical resection must be performed.

\section{Authors' affiliations}

X B D'Journo, V Moutardier, O Turrini, J Guiramand, B Lelong, C Pesenti, G Monges, M Giovannini, J R Delpero, Department of Surgical Oncology, Institut Paoli-Calmettes, 232 Boulevard de Sainte Marguerite, 13009 Marseille, France
Take home messages

- We report an adult case of gastric duplication cyst (GDC) mimicking a mucinous cystadenoma of the pancreas in which concentrations of carcinoembryonic antigen (CEA) and carbohydrate 19-9 (CA 19-9) were extremely high

- To our knowledge, this is the first report of a simultaneous increase in CEA and CA 19-9 in GDC in the absence of malignancy

- Although few cases of carcinoma arising from a GDC having been reported, the production of oncofetal antigens suggests the possibility of a precancerous condition, so that in this situation surgical resection must be performed

Correspondence to: $\operatorname{Dr} X$ B D'Journo, Institut Paoli-Calmettes, 232 Boulevard de Sainte Marguerite, 13009 Marseille, France; benoitdjourno@wanadoo.fr

Accepted for publication 10 June 2004

\section{REFERENCES}

1 Blinder G, Hiller N, Adler SN. A double stomach in an adult. Am J Gastroenterol 1999;94:1100-2.

2 Glaser C, Kuzinkovas V, Maurer C, et al. A large duplication cyst of the stomach in an adult presenting as pancreatic pseudocyst. Dig Surg 1998;15:703-6.

3 Ikehata A, Sakuma T. Gastric duplication cyst with markedly elevated concentration of carbohydrate antigen 19-9. Am J Gastroentero 2000;95:842-3.

4 Brugge WR, Lewandrowski K, Lee-Lewandrowski E, et al. Diagnosis of pancreatic cystic neoplasms: a report of the cooperative pancreatic cyst study. Gastroenterology 2004;126:1330-6.

5 Kuraoka K, Nakayama H, Kagawa T, et al. Adenocarcinoma arising from a gastric duplication cyst with invasion to the stomach: a case report with literature review. J Clin Pathol 2004:57:428-31.

6 Nomura T, Shirai Y, Hatakeyama K, et al. An adult ileal duplication cyst containing markedly elevated concentrations of carcinoembryonic antigen and carbohydrate antigen 19-9. Am J Gastroenterol 2002;97:208-9.

7 Madia $C$, Lumachi $F$, Veroux $M$, et al. Giant splenic epithelial cyst with elevated serum markers CEA and CA 19-9 levels: an incidental association? Anticancer Res 2003;23:773-6.

8 Tayar C, Brunetti F, Tantawi B, et al. Laparoscopic treatment of an adult gastric duplication cyst. Ann Chir 2003;128:105-8. 